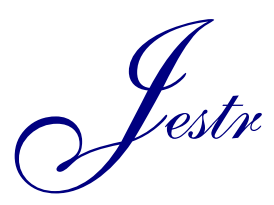

Journal of Engineering Science and Technology Review 12 (5) (2019) 25 - 30

Research Article

www.jestr.org

\title{
Ant Colony Based Mechanism for Increasing Life Time of Critical Nodes
}

\author{
J. Sandeep \\ Christ (Deemed to be University), Bangalore, India
}

Received 29 May 2018; Accepted 9 July 2019

\begin{abstract}
MANET nodes act as a host as well as a router which increases the significance of every node for their participation in communication. Loss of any node in the network results in failure of links connected to the node which brings the importance of increased lifespan of a node. Some nodes during frequent transaction at critical network scenario consume more energy and become ill with critical energy level. Special attention towards these nodes can improve the lifespan of the node. In this paper an ant colony-based pheromone deposition mechanism was proposed to extend the lifetime of ill nodes. Pheromone deposited for the neighbor in the pheromone table helps in identifying frequently communicated neighbor. The proposed algorithm identifies the ill node and requests its frequently communicated neighbor for a tie up. The neighbor shares the workload of the ill node with mutual agreement. This method also improves the performance of a network by limiting pheromone deposition practice for low weighted nodes with low energy and high density (packet in queue). The proposed method increases lifespan of ill nodes and thereby increases the lifetime of entire network. The proposed work was also compared with existing protocol and the results have proved that the proposed mechanism has increased lifetime and reduced energy consumption of the entire network.
\end{abstract}

Keywords: MANET, Routing, Ant Colony Optimization, Nodes Lifetime, Energy Efficiency

\section{Introduction}

Mobile Ad Hoc network (MANET) is a self-organizing network which benefits with its applications in infrastructureless environments Pelusi et al. [1]. This kind of network is well suited for the mission critical applications such as emergency relief, military operations where no predeployed infrastructure exists for communication Debdutta et al. [24]. The limitation of nodes reachability in the network influences into the enhancement of its role Motoyoshi et al. [3]. The device in this network participates as a host as well as intermediate for its transactions Renu et al. [4].

Nodes participation in the network differs based on its positioning with other nodes e.g. the central node in the network and nodes with more number of links have higher responsibilities in network transactions Khalid et al. [5], Abbas et al. [6]. The edge nodes on the other hand have less responsibility in the network Kampitaki et al. [8]. In a mobile environment, dynamic change in position results into frequent link failures Devi el at. [7] The link failures have high impact in the performance of the network. The major reason of frequent link failures has been identified as the uneven distribution of workload among nodes. Improving the energy efficiency of the nodes to get higher time to live (TTL) is a recent research demand in MANET and wireless sensor networks. This influences for the development of enhanced algorithms to control the responsibility distribution and increase the energy efficiency of nodes. Many researchers are continuously working in this area to improve network and nodes lifetime Mansourkiaia et al. [26], Tamandani et al. [27].

*E-mail address: mail2jsandeep@gmail.com

ISSN: 1791-2377 @ 2019 School of Science, IHU. All rights reserved.

doi:10.25103/jestr.125.03
Roberto Magan in his research article has presented a multicast routing based on tree topology which uses genetic algorithm as a computational stratergy to select optimal intermediate nodes. The approach uses maximum residual energy and minimal energy usage as its computational parameters in the selection of intermediate node [31]. Dimitris Kanellopoulos in his research focused on the transport layer and designed TCP enhancement for congestion control which in turn reduces energy consumption [29]. In a research work conducted by Hesham et al., DSR based scheme was introduced which finds power and load aware multiple node-disjoint paths to destination. The approach was able to show difference with little extension in the operational life of nodes [33]. In another approach, Anish $\mathrm{K}$ has modified the existing method with a planned and reduced usage of control message. This modified system uses transition state supporting cooperative MAC broadcast protocol. This method not only reduces the energy consumption but also utilizes the available node effectively before the nodes energy drains [30]. Farkhana Muchtar et al., conducted a detailed review to emphasize on the importance of energy efficiency in MANET. The research further explores with robust understanding of energy efficiency aspects in MANET [32].

The organization of this paper follows with problem identification and discussion in section 2. The next section discusses node and its state. The section also elaborates weight calculation and nodes categorization. The algorithms design and working process is detailed in section 4 . Experiment and simulation environment used in the network have been presented in section 5 this discusses the parameter used for the comparison and evaluation of protocol performance in the network. Section 6, presents the result 
with detail discussions. Finally, the conclusion was made based on the obtained results.

\section{Problem Identification}

Mobile ad hoc network has attracted more critical applications of medical emergencies, rescue and military operations Saju et al. [2]. Every data generated and shared between the nodes are vital and even carried out at the risk of human life. Each node in these application scenarios is significantly important for its service to the maximum. The situation can be analyzed from the example as shown in fig. 1. Network with seven wireless nodes (A, B, C, D, E, F and $\mathrm{G})$ have been shown in fig. 1. Line connecting nodes are the links of the network. Critical data is collected from the environment by Node $\mathrm{E}$ and distributes to the network. Low energy of the node makes it critically important to reduce its transaction workload El et al. [9] which in turn will increase the lifetime node. This can be made possible by increasing the cost of the link so that the route will be selected based on the link cost. The impact of increase in cost of links connecting the source of critical data will reduce the possibility of node to be selected as intermediate for networks transaction. The network will have a light weighted (High cost and low workload) links connecting such critical node.

In fig. 1, any case of route between $\mathrm{C}$ to $\mathrm{F}$ will be carried through $\mathrm{B}$, D neglecting the route through node $\mathrm{E}$ (as intermediate) in its first preference. It is also important not to block routes for critical requests in the network. Therefore, any repeated request increments its importance from normal to critical. The criticality gets increased every time the node receives duplicate request for the neglected request. This influences the node to allow critical request to form route through critical nodes. Identifying the critical node in the network is another task of this algorithm. Nodes energy, density and mobility were used to determine the function of routing protocols Jisha et al. [10] which are to categorize nodes current state. This categorization of nodes state separates the nodes under 3 groups, the efficient, busy and critical nodes.

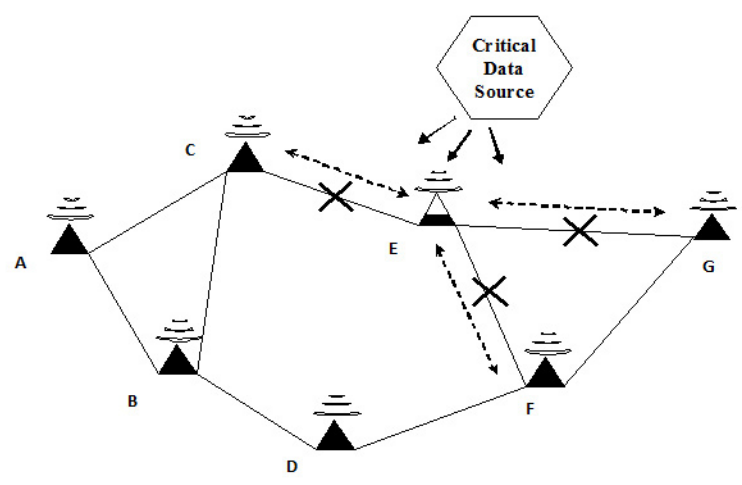

Fig. 1. Network scenario with critical node

The network needs to be efficiently monitored to identify its weak nodes. Nodes in a network were randomly spread with difference in location, movement and traffic. Effect of various mobile node movements pattern is analyzed in Saad et al. [11] and shows the impact in networks performance. These differences and the frequency with which the node participates in communication will dynamically affect its state. Frequently used nodes will have more traffic and consumes more energy compare to other nodes. The proposed method utilizing the frequently used path to reach the destination and even finds the best path with higher deposition of pheromone by its backward packets (reply packet). It was analyzed that less active nodes had no advantage of collecting such pheromone information as the pheromone is collected and evaporated without being utilized. In any network the participating nodes go through different state of life. To make the best use of optimization it needs to be identified for which state of nodes the optimization benefits more and reducing the overhead from other nodes. In fig. 2 the network highlights the busy node based on its traffic. Energy consumption of entire network can be reduced, if selected nodes execute additional activity (using the frequently used information) to optimize its control overhead. The proposed work identifies busy nodes to efficiently utilize the advantages of frequently used routes and ant colony optimization. The network to identify weak node were frequent monitored for every nodes state.

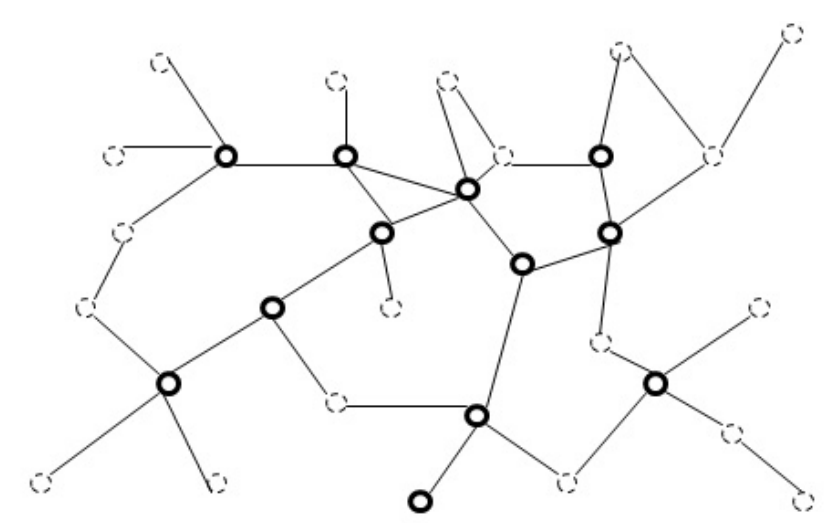

Fig. 2. Network with busy and efficient nodes.

Many algorithms have been proposed in literature which monitors the network and its state [25][28]. The algorithm proposed in this work, identifies the three different state of the node and allows executing different approach at nodes based on their current state.

\section{State of Node}

Network is formed with two or more nodes exchanging information and participating as intermediate Jahangir et al. [12], Tejomayee et al. [13], Djamel et al. [14] in networks transactions. As from the problem definition network needs to identify three different categories of nodes.

\section{1) Efficient Nodes \\ 2) Busy Nodes \\ 3) Critical Nodes}

Nodes based on their weight were categorized into these three categories. The weight is calculated for nodes residual energy, density of packets in the queue and nodes movement Jihen et al. [15], Hsiu et al. [16].

\section{Weight Calculation and Determining Nodes State}

In the proposed approach, every node evaluates its own weight and updates its neighbor for any change in its current state. The algorithm uses Fuzzy system to identify the state of 
the node. A triangular membership function is defined as a function having three points in the variation range of a parameter. The triangular membership function can be mathematically represented as Eq.1 and Eq.2.

$\succ(x ; a, b, c)=\left\{\begin{array}{c}0, \text { if } x<a \\ \frac{x-a}{b-a}, \text { if } a \leq x<b \\ \frac{c-x}{a-b}, \text { if } b \leq x<c \\ 0, \text { if } c \leq x\end{array}\right.$

The above equation can be written in the compact form as

$$
\succ(x ; a, b, c)=\max \left(\min \left(\frac{x-a}{b-a}, \frac{c-x}{a-b}, 0\right)\right)
$$

Where $\mathrm{x}$ is an input vector, and $\mathrm{a}, \mathrm{b}, \mathrm{c}$ are number which were obtained by measurements. Fuzzy set and fuzzy operations are acts and actuators in fuzzy logic. There are three input variables mobility, battery life and processing density in the fuzzy reasoning system. Tab. 1 presents the triangular membership function used in the experiment to convert the crisp input to fuzzy. The mobility, density and battery life vary from 0 to $100 \%$ three membership functions for these input variables were low, medium and high been assigned as shown in tab. 1. Output variable, weight value, varies from 0 to 100 units. Three membership functions for its variables were considered as low, medium and high. The if-then rule formulizes the necessary conditions for fuzzy logic to decide the grading. The inference mechanism used in the experiment consists of 27 rules as shown in tab. 2 .

\section{Fuzzy Inference Mechanism}

If and then rule was used in the system to identify the fuzzy output for the given fuzzy input Budyal et al. [17], Hongjun et al. [18], ParthaSarathi et al. [19]. The following rules were used to establish a relationship between the input and output variables. These rules were obtained through the primary experiments using NS2 simulation with different scenarios. Tab. 2 shows the association of fuzzy input to get the weight of the node. Center of gravity method has been used in the defuzzification of output variable.

Table 1. Fuzzy Triangular Membership Function to Convert Crisp Inputs to Fuzzy

\begin{tabular}{l|c|l|c}
\hline \multicolumn{1}{c|}{ Density } & Parameters & Battery & Parameters \\
\hline Low & 0040 & Low & 0040 \\
Medium & 105090 & Medium & 105090 \\
High & 60100100 & High & 60100100 \\
\hline \multicolumn{1}{c}{ Mobility } & Parameters & Weight & Parameters \\
\hline Low & 0040 & Low & 0040 \\
Medium & 105090 & Medium & 105090 \\
High & 60100100 & High & 60100100 \\
\hline
\end{tabular}

Table 2. Fuzzy Rules for Nodes Weight

\begin{tabular}{|c|c|c|c|}
\hline \multirow{2}{*}{$\begin{array}{l}\text { Mobility / } \\
\text { Density }\end{array}$} & \multicolumn{3}{|c|}{ Weight@ } \\
\hline & $\begin{array}{c}\text { Low } \\
\text { Energy }\end{array}$ & $\begin{array}{c}\text { Medium } \\
\text { Energy }\end{array}$ & $\begin{array}{c}\text { High } \\
\text { Energy }\end{array}$ \\
\hline Low / Low & Medium & Low & Low \\
\hline Low / Medium & Medium & Low & Low \\
\hline Low / High & High & Medium & Medium \\
\hline Medium / Low & Medium & Low & Low \\
\hline
\end{tabular}

\begin{tabular}{|c|c|c|c|}
\hline $\begin{array}{l}\text { Medium / } \\
\text { Medium }\end{array}$ & High & Medium & Low \\
\hline $\begin{array}{l}\text { Medium / } \\
\text { High }\end{array}$ & High & Medium & Medium \\
\hline High / Low & High & Medium & Medium \\
\hline $\begin{array}{l}\text { High / } \\
\text { Medium }\end{array}$ & High & Medium & Medium \\
\hline High / High & High & High & Medium \\
\hline
\end{tabular}

Consequently, weight of each node has been calculated to grade current state of node.

\section{Design and Steps of Algorithm}

The proposed work, named as Enhanced ANT (E-ANT) was designed to optimize networks performance by categorizing the nodes state and optimizing candidate nodes control overheads to improve their life time. Step by step process of the algorithm is discussed as follows. Any node on receiving a route request (FPkt(id)), it updates request priority for the packet id where every duplicate request increments its processing priority. If there is no other route exists in the network, the priority is useful in identifying the criticality of the request. On receiving the request packet, the node checks its current state (Critical, Busy \& Efficient) and responds to the request based on its current state. Algo. 1 presents the request packet and its possible response sequences in the proposed work.

In the proposed work ant colony-based pheromone deposition was made for the busy and critical nodes. Every route request and reply packet from the neighbor is updated by the node. The algorithm responds to the reply packet by forwarding the packet to its route. If the node finds itself as critical to respond to this packet it drops the packet. The pheromone deposited gets evaporated at Pt time interval to remove old data. Algo.2 presents the algorithm with steps that is executed for the reply packet.

\section{Frequency and Time Interval for Monitoring Nodes State}

The network needs to update every change that may affect in the performance to enable error free transactions Trung et al. [20]. The refresh algorithm helps the network to update the changes cause by the movement of node, networks energy flow and traffic. In the proposed approach, network executes (Triggers) refresh algorithm in the cause of following events.

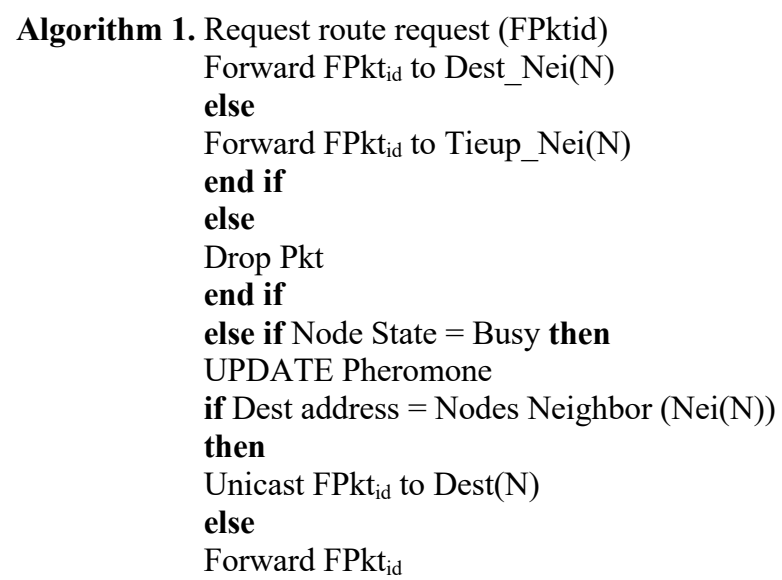




\section{end if}

else if Node State $=$ Efficient then

if Dest address $=$ Nodes Neighbor $(\operatorname{Nei}(\mathrm{N}))$

then

Unicast $\mathrm{FPkt}_{\mathrm{id}}$ to $\operatorname{Dest}(\mathrm{N})$

else

Forward $\mathrm{FPkt}_{\mathrm{id}}$

end if

end if

- Nodes mobility change at high rate $(\mathrm{MH})$

- Nodes Energy drop with high rate (EH)

- Higher packet rate in the queue (PH)

Algorithm 2. Receives route reply (RPktid)

RPktid Priority $=$ RPktid Priority +1

if Source address $=$ Node itself $(\mathrm{N})$ then

Establish Connection

else if Node State $=$ Critical then

Update Pheromone

if RPktid Priority High then

Unicast RPktid to $\mathrm{Next}$ Nei(N)

else

Drop PKT

end if

else if Node State $=$ Busy then

UPDATE Pheromone

Unicast RPktid to Next Nei(N)

else if Node State $=$ Efficient then

Unicast RPktid to Next Nei(N)

end if

Algorithm 3. Refresh nodes state

$\{\mathrm{Nm}$ : Nodes Mobility; Pktr: Pkt rate at queue;

Ec: Change in energy\}

if time $=\mathrm{T}\left\|\mathrm{Nm}{ }_{-} \mathrm{MH}\right\| \mathrm{Pktr} \_=\mathrm{PH} \| \mathrm{Ec} \_\mathrm{EH}$

then

Calculate Nodes State

\{Before Refresh\}

Before State $=$ Current Node State

INPUT: Nodes Energy, Nodes Density, Nodes Mobility

PROCESS: Fuzzy system, Fuzzification,

Inference Rules, Defuzzification

\{After refresh\}

OUTPUT: Node State

if Before state $=$ Busy $\|$ Efficient \&\& After

State $=$ Critical then

Send tieup Request to highest priority

neighbor

if Accepted then

Add node as tieup attainder node

Goto Sleep Mode

else

Send request to next

end if

else if Before state $=$ Critical \&\&After State $=$

Busy||Efficient then

Free the tied-up node

Send update to neighbor nodes

end if

end if
In addition to all three events there will be a constant time interval $\mathrm{T}$ at which the network executes refresh algorithm. The algorithm keeps a check on to the state of the network nodes which improves the reliability of the network at any point of time. Nodes at high traffic with more packets in there queue causes delay in networks transaction and even in cases it drops packet at high dense situations. Node with high mobility results into frequent change in topology therefore nodes movement has been considered while monitoring the state of any node. Energy of the node is one of the major limited resources of MANET Baisakh et al. [21], Adam et al. [22], Afzal et al. [23] that have been taken as the third variable to evaluate the state of node.

\section{Simulation Environment}

The work has been simulated to study the performance of proposed algorithm. As the objective of the work was to identify the weak nodes in the network and reduce their workloads to increase nodes lifespan. Experimental setup was made with 50 to 100 nodes network. Each node was assigned with random energy values (ranging between 50 joules to 100 joules). Network was provided with high traffic to generate network scenario where few or some nodes come across the risk of becoming weak nodes. Other parameters that were set for the experiment are detailed below in the tab. 3 .

Table 3. Simulation Parameters

\begin{tabular}{l|l}
\hline \multicolumn{1}{c|}{ Parameters } & \multicolumn{1}{c}{ Values } \\
\hline NS-2 Version & 2.35 \\
Area in sq mtrs & 1000 \\
Number of Nodes & $50-160$ \\
MAC layer & 802.11 \\
Packet Size & 2000 bytes \\
Interval & $0.25 \mathrm{~ms}$ \\
Packet Rate & $4 \mathrm{pkt} / \mathrm{s}$ \\
Traffic \& Transport Protocol & $\mathrm{CBR}:$ FTP \& UDP : TCP \\
Bandwidth & $2 \mathrm{Mbits} / \mathrm{Sec}$ \\
Antenna & Omni antenna \\
Radio Propagation & Two ray ground \\
Transmission Energy & $2 \times 10-1$ joules/pkt \\
Receiving Energy & $1 \times 10-1$ joules/pkt \\
\hline
\end{tabular}

\section{Result and Discussion}

Simulation for the proposed approach was carried out in two different experimental environments. In the first set of studies the proposed approach was compared with AODV protocol for the impact of mobility. In the second set of experiment the proposed method was studied at varying traffic loads. Packet interval from the source node was varied and the proposed method was compared with ANT based routing approach.

Results obtained from the mobile environment were presented in fig. 3 and fig. 4. At high mobility the packet delivery ratio for the E-ANT was $99.93 \%$ and AODV was able to deliver $94.623 \%$ of packets as shown in fig. 3 . The plot further shows that the proposed E-ANT protocol has consistently given higher packet delivery ratio at different mobility environment. High mobility has higher impact on AODV protocol in terms of its packet delivery performance. The packet delivery ratio for AODV protocol was approximately $96.9 \%$. 


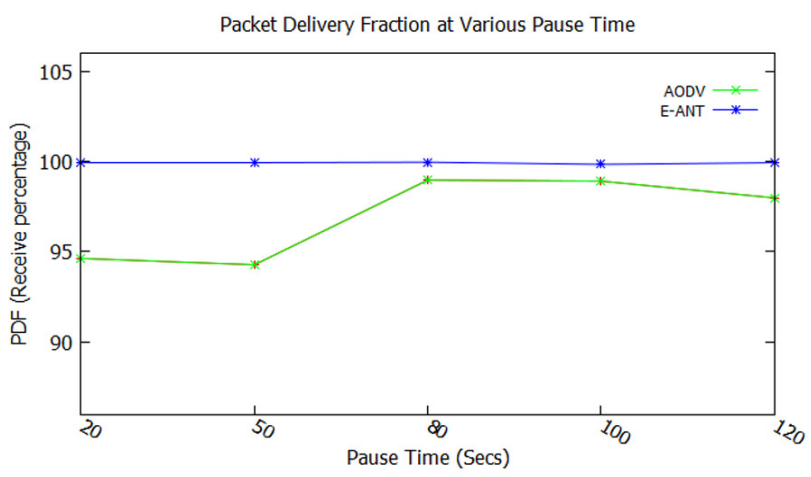

Fig. 3. PDF at various pause time.

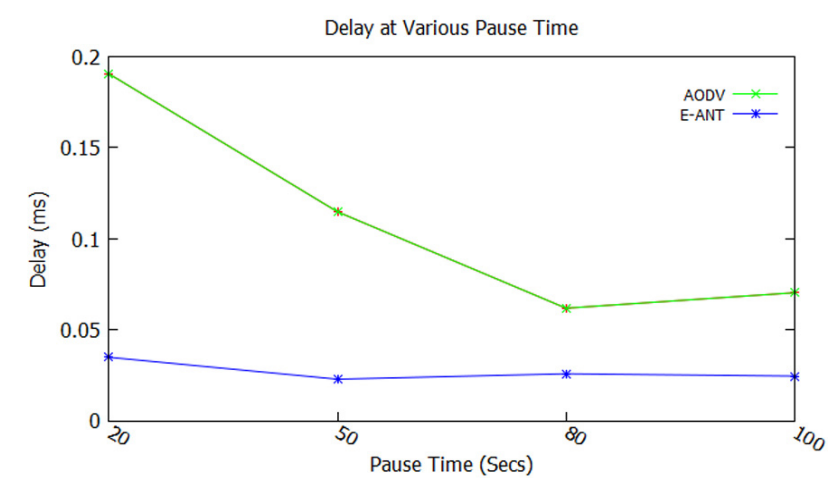

Fig. 4. Delay at various pause time

In Fig. 4, simulation result for delay in the network was compared for E-ANT and AODV protocol. The results prove that E-ANT has less delay compared to AODV at different pause time.

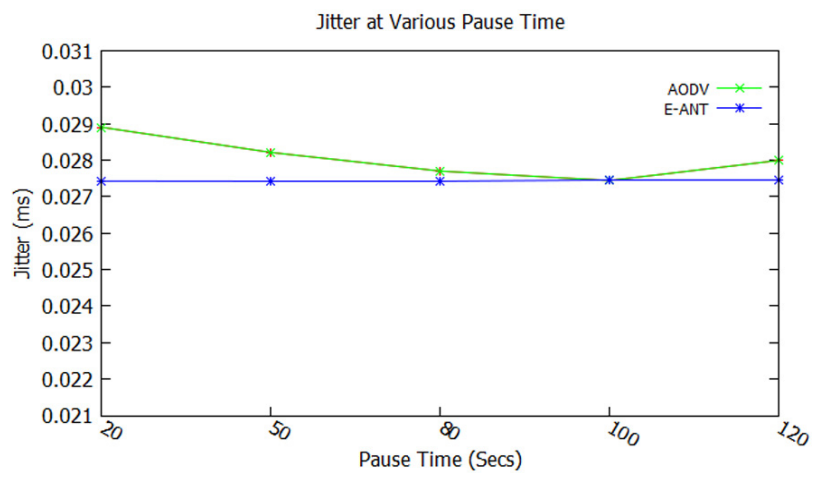

Fig. 5. Jitter at various pause time.

Figure 5 presents the jitter in the network for E-ANT and AODV at various pause times. It can be observed from the graph that the proposed method E-ANT had less jitter from higher mobility to lower mobility.

Simulation results for varied packet interval from source were presented in fig. 6 and fig. 7. The results show that high traffic has higher impact over the performance of routing approaches. Fig. 6 presents the comparison of proposed approach with ANT routing algorithm. At packet interval of $0.05 \mathrm{~ms}$, proposed approach was able to deliver $53.81 \%$ of packet whereas ANT had low packet delivery fraction. The reduced traffic with a packet interval of $0.08 \mathrm{~ms}$ and $0.1 \mathrm{~ms}$ has allowed the protocols to deliver packets at higher rate of above $90 \%$. Throughout the Enhance ANT has performed higher with improved packet delivery ratio.

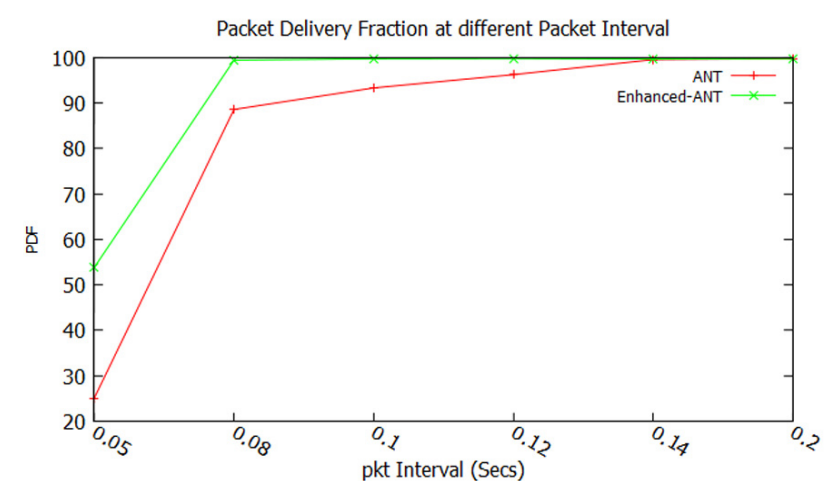

Fig. 6. PDF at different packet intervals

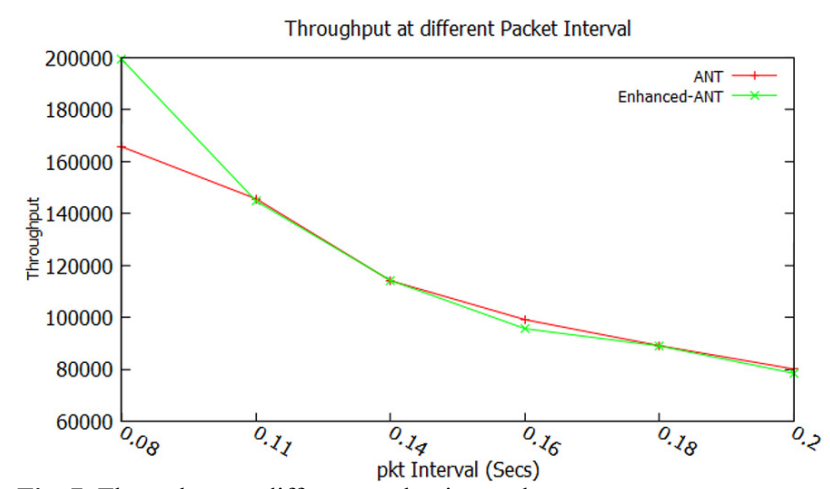

Fig. 7. Throughput at different packet intervals

In fig. 7 the simulation results for the ANT and Enhance ANT were compared for throughput in the network. Results show that the proposed approach had higher throughput for very low packet intervals but this difference in throughput was very moderate for average and higher intervals. In the results the jitter observed for the E-ANT was low when compared with ANT protocol.

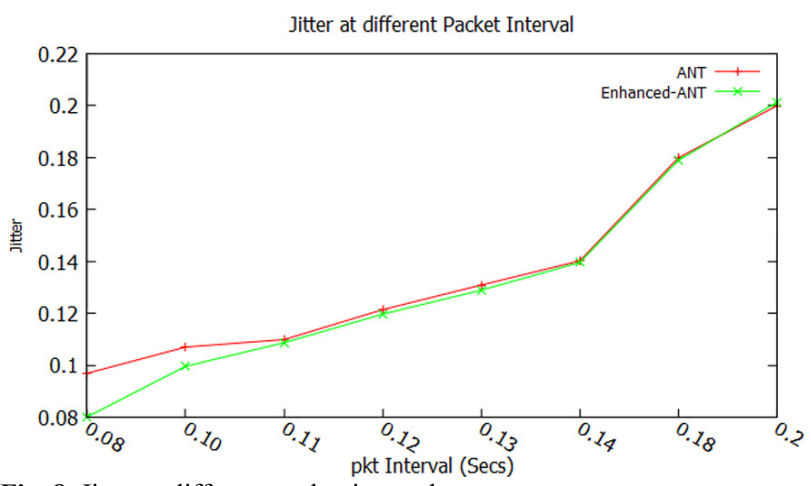

Fig. 8. Jitter at different packet intervals

Fig. 8. presents the jitter in network for ANT and E-ANT at varying packet interval.

\section{Conclusion}

This work was concert with the critical nodes in mobile ad hoc network. There was a need to enhance the performance of such critical node and networks performance. In this approach, ANT colony-based approach was enhanced with the awareness of nodes state and simulated. The results have proved the improved performance of E-ANT at various mobility and traffic scenarios. The proposed approach was able to improve the packet delivery fraction at an approximate range of $99.93 \%$ for different mobility scenarios and 
approximate $99.7 \%$ of PDF at traffic scenarios. The study has also shown reduce in delay and jitter for the proposed approach when compared.
This is an Open Access article distributed under the terms of the Creative Commons Attribution License

\section{References}

[1] Pelusi, L., Passarella, A., Conti, M. Opportunistic Networking: Data Forwarding in Disconnected Mobile ad hoc Networks. IEEE Communication Magazine, vol. 44, issue 11, pp. 134 - 141, (2006).

[2] Saju, P.J., \& Philip, S. Self-Organized Key Management with Trusted Certificate Exchange in MANET. Ain Shams Engineering Journal, Science Direct, vol. 61, pp. 161-170, (2015).

[3] Motoyoshi, G., Leibnitz, K., \& Murata, M. Mobility Tolerant Firework Routing for Improving Reachability in MANETs. Future Internet, vol. 6, pp. $171-189$, (2014).

4

[5] Khalid, H., Abdullah, A.H., Saleem, I., Khalid, M.A., \& Faraz, A. Efficient Cluster Head Selection Algorithm for MANET. Journal Of Computer Networks And Communications, Hindawi Publishing Corporation, vol. 2013, pp. $01-07$, (2013).

[6] Abbas, K., Afsharfarnia, A., Zarafshan, F., \& Haddad, S.A.R Al Haddad. A Novel Clustering Algorithm For Mobile Ad Hoc Networks Based On Determination Of Virtual Links' Weight To Increase Network Stability. The Scientific World Journal, vol. 2014, pp. $01-11,(2014)$.

[7] Devi, M., \& Rhymend, U.V. Particle swarm optimization (PSO)based node and link lifetime prediction algorithm for route recovery in MANET. EURASIP Journal on Wireless Communications and Networking, 2014, pp. 01 - 10, (2014).

[8] Kampitaki, D., \& Economides, A.A. Simulation Study Of MANET Routing Protocols Under Ftp Traffic. Procedia Technology, Elsevier, vol. 17 , pp. $231-238,(2014)$.

[9] Le, G., Shankar, M.B., \& Lau, C.N. Managing Real-Time Database Transactions in Mobile Ad-Hoc Networks. Distributed and Parallel Databases, Springer US, vol. 22, issue 1, pp. 27 - 54, (2007).

[10] Jisha, G., \& Philip, S. Impact of Node Density on Node Connectivity in MANET Routing Protocols. Advances in Computing and Communications. Communications in Computer and Information Science, vol. 193, pp. $01-08,(2011)$.

[11]Izuan Mohd Saad, Mohd \& Zukarnain, Zuriati. Performance Analysis Of Random Based Mobility Models in MANET Routing Protocol. European Journal of Scientific Research, vol. 32 issue 4, pp. $444-454,(2009)$.

[12] Jahangir, K., Hyder, S.I., FakarDuani, S.M., \& Mustafa, S. Modeling and Simulation of Dynamic Intermediate No Des and Performance Analysis in MANETs Reactive Routing Protocols. International Journal on Distributed Computing, vol. 4, issue 1, pp. $31-56$, (2011).

[13] Tejomayee, N., \& Suneeta, M. An Efficient Routing Approach and Improvement of AODV Protocol in Mobile Ad-Hoc Networks. International Journal of Computer Science and Information Technologies, vol. 2, issue 3, pp. 1099 - 1104, (2011).

[14]Djamel, D., Abdelouahid, D., \& Nadjib, B. Adhoc Networks Routing Protocols Mobility. The Arab Journal of Information Technology, vol. 3, issue 2, pp. 126-133, (2006).

[15]Jihen D.R., Leila, B., \& Henda, B.G. Performance Evaluation and Impact of Weighting Factors on An Energy And Delay Aware Dynamic Source Routing Protocol. International Journal of Computer Science \& Information Technology, vol. 3, issue 4, pp. 225 $-244,(2011)$.

[16]Hsiu P.C., \& Kuo, T.W. A Maximum-Residual Multicast Protocol For Large- Scale Mobile Ad Hoc Networks. IEEE Transactions on Mobile Computing, vol. 8, issue 11, pp. 1441 - 1457, (2009).
[17] Budyal, V.R., \& Manvi, S.S. Intelligent Agent Based Delay Aware QOS Unicast Routing In Mobile Ad Hoc Networks. International Journal of Multimedia and Ubiquitous Engineering, vol. 8, issue 1, pp. $11-28$, (2013).

[18]Hongjun Dai, Jia Z., \& Qin Z. Trust Evaluation and Dynamic Routing Decision Based on Fuzzy Theory for MANETs. Journal of Software, vol. 4, issue 10, pp. 1091-1101, (2009).

[19] Parthasarathi B., Paulchoudhury, J., \& BhadraChaudhuri, S.R. Fuzzy Membership Function in a Trust Based AODV for MANET. International Journal of Computer Network and Information Security, vol. 12, pp. $27-34$, (2013).

[20] Trung, K.V., \& Sungoh, K. Mobility-Assisted On-Demand Routing Algorithm for MANETs in the Presence of Location Errors. The Scientific World Journal, vol. 2014, pp. $01-11,(2014)$.

[21] Baisakh., Patel, N.R., \& Kumar, S. Energy Conscious DSR in MANET. In Proceedings of International Conference On Parallel Distributed And Grid Computing (Pdgc), IEEE, pp. 784 - 789, (2012).

[22] Adam S.M., \& Rosilah, H. Delay Aware Reactive Routing Protocols For QOS In MANETs: A Review. Journal of Applied Research and Technology, vol. 116, pp. 844 - 850, (2013).

[23] Afzal, M., \& Hossam, H. Efficient Content Distribution for Peer-ToPeer Overlays on Mobile Ad Hoc Networks. Journal of Advanced Research, vol. 2, pp. 265 - 279, (2011).

[24]Debdutta, B.R., \& Rituparna, C. MADSN: Mobile Agent Based Detection of Selfish Node in MANET. International Journal of Wireless \& Mobile Networks, vol. 34, pp. 225 - 235, (2011).

[25] Iqbal, M., Gondal, I., \& Dooley, L.S. Optimizing Network Control Traffic in Resource Constrained MANETs. In the Proceedings of TENCON 2005, Melbourne, Qld., IEEE, pp. 1 - 7, (2005).

[26] Mansourkiaie F et al. Maximizing Lifetime in Wireless Sensor Network for Structural Health Monitoring with and without Energy Harvesting. Special Section on Energy Harvesting and Scavenging: Technologies, Algorithms, And Communication Protocols, IEEE, vol.5, pp. $2383-2395$, (2017).

[27] Tamandani Y K et al. Computing geometric median to locate the sink node with the aim of extending the lifetime of wireless sensor networks. Egyptian Informatics Journal. vol. 18, Issue 1, pp. 21-27, (2017).

[28] Liu X et al. Distributed cooperative communication nodes control and optimization reliability for resource-constrained WSNs. Neurocomputing, Elsevier, vol. 270, pp. 122-136, (2017).

[29]Dimitris Kanellopoulos. Congestion control for MANETs: An overview. ICT Express, vol. 5, Issue 2, pp. 77-83, (2019)

[30] Anish K, Pon Yamini, Suthendranb K and Arivolic T. Enhancement of energy efficiency using a transition state mac protocol for MANET. Computer Networks, vol. 155, pp. 110-118, (2019).

[31] RobertoMagan Carrion et al. A Dynamical Relay node placement solution for MANETs. Computer Communications, vol. 114, pp. 3650, (2017).

[32] Farkhana Muchtar et al. Energy conservation strategies in Host Centric Networking based MANET: A review. Journal of Network and Computer Applications, vol. 111, Issue 1, pp. 77-98, (2018).

[33] Hesham A.Ali et al. An on-demand power and load-aware multi-path node-disjoint source routing scheme implementation using NS-2 for mobile ad-hoc networks. Simulation Modeling Practice and Theory. vol. 80, pp. 50-65, (2018). 